\title{
Gravitational Lensing and Cusp Slope Value Limit for the Dark Matter Haloes
}

\author{
Petri Mutka* \\ University of Oulu \\ E-mail: Petri.Mutka@oulu.fi
}

We have developed an analytical theory for axially symmetric lensing with the generalized Navarro-Frenk-White density profile (GNFW). The density profile is strongly cusped at the center, i.e. when $r<<1$, density $\rho \propto r^{\alpha}$, where $\alpha=[-2,-1)$. We apply our lensing theory to the statistical study of double-image quasar lenses. The lensing model gives a relation between location of the optical axis, the cusp slope and the magnification ratio of the images. It does not depend directly on the cosmology, the distance of the lens, the distance of the source nor the mass of the lens object. We use this relation to derive the statistics of the upper limits for the cusp slopes, which are capable producing the observed magnification ratios for each lens. The composition of the distribution depends on the general properties of the dark matter haloes in the lens system sample.

The statistics is affected by several error sources; the lens ellipticity, the variability of the source coupled with the time delay effects, and the perturbations by the substructure within the lens. Here we study the effects of the substructure within the lensing potential on the overall statistics of the cusp slope limits.

The Manchester Microlensing Conference: The 12th International Conference and ANGLES Microlensing Workshop

January 21-25, 2008

Manchester, $U K$

* Speaker. 


\section{Background}

We assume that the density profile of dark matter halo is described by the GNFW profile (Navarro et al. 1996; Zhao 1996; Navarro et al. 1997). The lens equation of a GNFW halo with a cusp slope $\alpha=[-2,-1)$ becomes particularly simple when it is normalized with the Einstein radius of the lens (Mutka \& Mähönen 2006):

$$
l=\left\{\begin{array}{ll}
q k\left(1-|k|^{\alpha+1}\right), & \text { when } k \leq k_{B} \\
k-\frac{\alpha+1}{\alpha+3} \frac{k_{B}^{2}}{k}(1-q), & \text { when } k>k_{B}
\end{array} .\right.
$$

Here $k$ is a radial coordinate at the lens plane and $l$ corresponding coordinate at the source plane. Information on the cosmology, the lens distance, the source distance, the mass and the concentration of the lens object are embedded in the constant $q$.

The lens equation (1.1) has a piecewise definition, that is divided at

$$
k_{B}=\left(\frac{2(q-1)}{q(\alpha+3)}\right)^{1 /(\alpha+1)}
$$

in order to avoid negative surface densities. Because $q>1$ by it's definition (Mutka \& Mähönen 2006), it is easy to see that $k_{B}>(2 /(\alpha+3))^{1 /(\alpha+1)}>k_{c r 1}$ holds always. Thence the maximum source coordinate $l_{\max }$ for strong lensing can be solved by setting $k=k_{c r 1}=(\alpha+2)^{-1 /(\alpha+1)}$ in the lens equation (1.1).

In general, for strong lensing it holds

$$
\frac{\left|k_{1}\right|^{\alpha+2}+\left|k_{2}\right|^{\alpha+2}}{\left|k_{1}\right|+\left|k_{2}\right|}=1
$$

for the solutions of images $k_{1}$ and $k_{2}$ when $k_{1}, k_{2}<k_{B}$. Equation (1.3) holds most of the time, and it is broken only with large lens masses or concentrations, i. e. $k_{1}$ or $k_{2}$ exceeds the value $k_{B}$. With the lens equation (1.1) and relation (1.3), the magnification ratio $\mu_{1} / \mu_{2}$ of the source at $l$ producing images at $k_{1}$ and $k_{2}$ can be written as

$$
M=\frac{\mu_{1}}{\mu_{2}}=\theta \frac{\left|\theta^{\alpha+2}+1-(1+\theta)(\alpha+2)\right|}{\left|\theta^{\alpha+2}+1-\theta^{\alpha+1}(1+\theta)(\alpha+2)\right|} .
$$

Note that constant $q$ has vanished from this expression. It is also possible to parametrize the lens equation with coordinate ratio $\theta=k_{1} / k_{2}$.

A solution $\alpha=\alpha_{C S L}$ and $\theta=\theta_{C S L}$ for the following pair of equations

$$
\left\{\begin{array}{l}
\frac{\mathrm{d} M}{\mathrm{~d} \theta}=0 \\
M=M_{0}
\end{array}\right.
$$

gives a CSL limit for the lens system. Here $M_{0}$ is the measured magnification ratio of the lens system. The value $\alpha_{C S L}$ is the minimum amount of "cuspiness", i.e. the maximum value for $\alpha$, that is needed to produce the observed magnification ratio $M_{0}$ with any lensing geometry assuming axial symmetry. A remarkable property of the CSL-value is that no information about the location of the mass center nor the optical axis of the lens system is required (Mutka \& Mähönen 2008).

The statistics of $\alpha_{C S L}$ values are constructed by assuming that the source images $((l / q)<$ $\left.(l / q)_{\max }\right)$ are uniformly distributed at the source plane. If all the lens systems in the sample have the same universal cusp slope value, a distinctive distribution of $\alpha_{C S L}$ values is produced, as presented in figure 1. If the cusp slopes of the halos are randomly distributed, this feature is destroyed. 

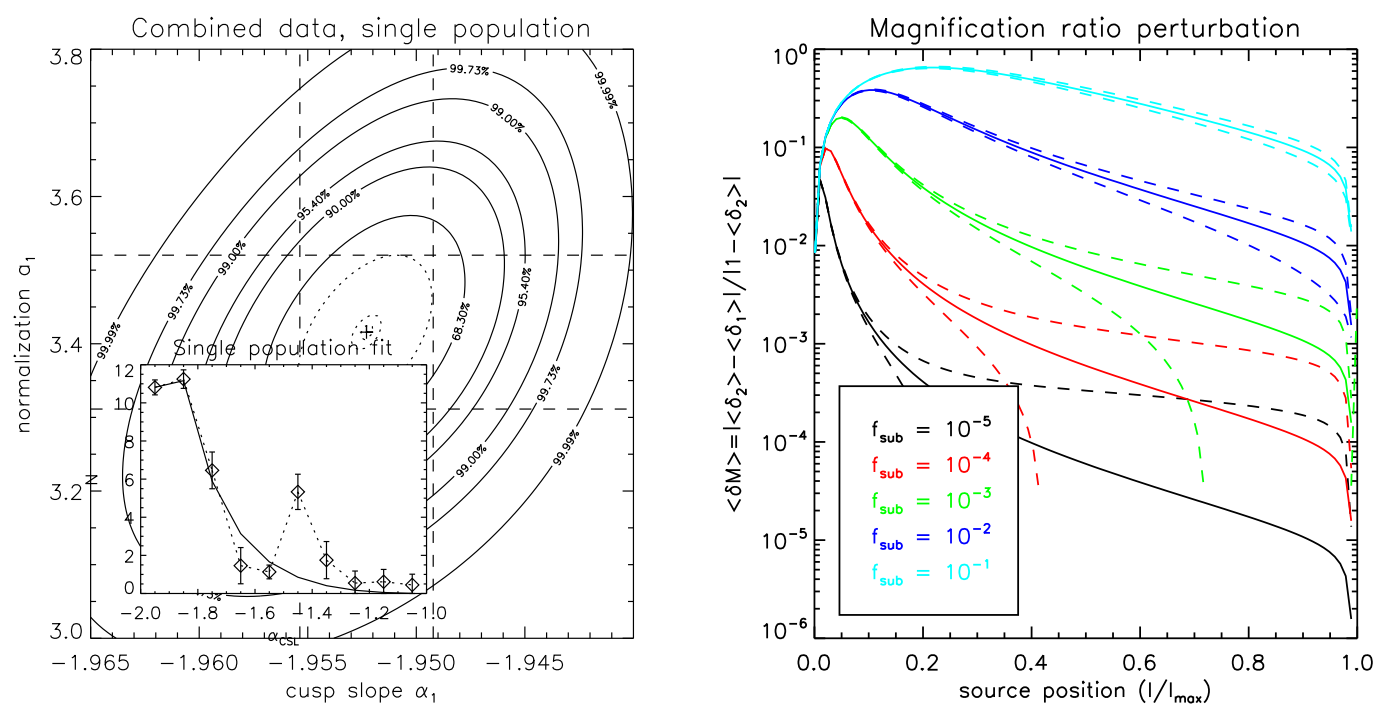

Figure 1: Left panel: CSL-distribution for a sample of known double-image quasar lenses and the best fit for the distribution. The exponential slope in the observations is suggesting that there is a population of lenses with a universal cusp slope value $\alpha \sim-1.95$. Right panel: Expectation value $\langle\delta M\rangle$ for perturbations of the magnification ratio with different amounts of substructure $f_{\text {sub }}$. Corresponding dashed curves indicate standard deviation of the normal distribution $\left(z_{l}=0.3, z_{s}=1.5, M_{l}=10^{12} M_{\odot}\right)$

\section{Substructure}

The effects of the substructure on the magnification of images is modelled with perturbations $\delta=\delta \mu / \mu$ that have normal distribution with expectation value $\langle\delta\rangle$ and variance $\operatorname{var}(\delta)$. It is assumed that $|\delta| \ll 1$, thus the astrometric perturbations by the substructure are assumed being negligible and only the changes in the fluxes of the images are considered. The moments of the distribution for the perturbations are calculated from the linear theory (Rozo et al. 2006).

Mass spectrum $s$ of the SIS perturbers is assumed to follow $\mathrm{d} s / \mathrm{d} m \propto m^{\beta}$, where $\beta=-1.8$ (Gao et al. 2004). The amount of substructure is defined by a ratio of the substructure surface density to the critical density $f_{\text {sub }}=2 \Sigma_{s} / \Sigma_{c}$. The other parameter is the cutoff of the mass spectrum $m_{\max }$ that is chosen to be $1 \%$ of the virial mass of the lens object.

\section{The synthetic lens catalogue}

The effects of the substructure on the CSL analysis are studied by constructing mock lens catalogues. The Press-Schechter function is sampled at the suitable range for the lens and the source objects. The luminosity of the source object is related to the sampled mass for the source (Wyithe \& Loeb 2002). The effects of the duty cycle time of quasars are ignored.

The magnification and the image separation biases are introduced by solving the lens equation (1.1) for the system with the random perturbations by the substructure. The resulting lens system is accepted if it exceeds the threshold magnitude and the necessary image separation. The effects 


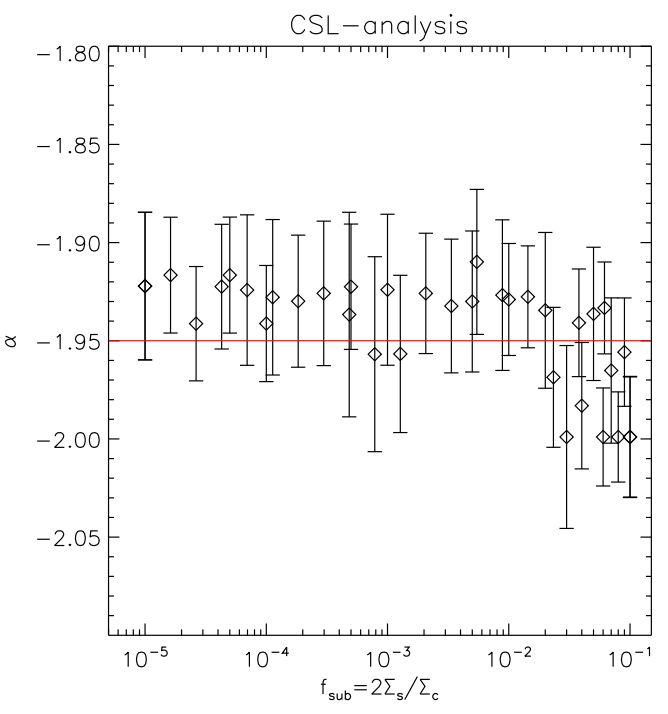

Figure 2: Recovered cusp slope values as a function of the substructure $f_{\text {sub }}$ in the lenses. Each point represents a catalogue of 100 lens systems with $\alpha=-1.95$ (red line). Error bars represent 0.1 magnitude accuracy in the flux measurements and errors in fitting. Weighted mean of trials is $\alpha=-1.946 \pm 0.005$.

of the substructure in the halos is studied by performing the CSL analysis on the mock catalogues with varying amounts of the perturbations $f_{\text {sub }}$.

\section{Conclusions}

The mock catalogues were produced with the cusp slope value $\alpha=-1.95$, and the values were recovered through the CSL analysis with moderate accuracy in all the cases. Strengthening the substructure seems to bring the recovered value closer to the $\alpha=-2.0$, which corresponds to the SIS model for the macrolens.

The amount of the substructure needed destroying the characteristic distribution for a universal cusp slope is out of range of the linear perturbation theory. At the nonlinear regime the perturbers affect the convergence and the shear of the macrolens thereby changing it's solution as well. That is a more challenging problem exceeding the scope of this presentation.

\section{References}

Mutka, P. T. \& Mähönen, P. H., 2006, MNRAS, 373, 243.

Mutka, P. T. \& Mähönen, P. H. 2008, manuscript in preparation.

Gao et al. ,2004 MNRAS, 355, 819.

Navarro, J. F., Frenk, C. S. \& White, S. D. M. 1996, ApJ, 462, 563.

Navarro, J. F., Frenk, C. S. \& White, S. D. M. 1997, ApJ, 490, 493.

Rozo, E., Zentner, A. R., Bertone, G. \& Chen, J. 2006, ApJ, 639, 573.

Wyithe, J. S. B. \& Loeb, A. L. 2002, ApJ, 581, 886.

Zhao, H. S. 1996, MNRAS, 278, 488. 